\title{
Modeling the Effect of Hyporheic Mixing on Stream Solute Transport
}

DOI:

10.1029/2019WR025697

\section{Document Version}

Accepted author manuscript

Link to publication record in Manchester Research Explorer

\section{Citation for published version (APA):}

Bottacin Busolin, A. (2019). Modeling the Effect of Hyporheic Mixing on Stream Solute Transport. Water

Resources Research . https://doi.org/10.1029/2019WR025697

\section{Published in:}

Water Resources Research

\section{Citing this paper}

Please note that where the full-text provided on Manchester Research Explorer is the Author Accepted Manuscript or Proof version this may differ from the final Published version. If citing, it is advised that you check and use the publisher's definitive version.

\section{General rights}

Copyright and moral rights for the publications made accessible in the Research Explorer are retained by the authors and/or other copyright owners and it is a condition of accessing publications that users recognise and abide by the legal requirements associated with these rights.

\section{Takedown policy}

If you believe that this document breaches copyright please refer to the University of Manchester's Takedown Procedures [http://man.ac.uk/04Y6Bo] or contact uml.scholarlycommunications@manchester.ac.uk providing relevant details, so we can investigate your claim.

\section{OPEN ACCESS}




\title{
Modeling the Effect of Hyporheic Mixing on Stream Solute Transport
}

\author{
Andrea Bottacin-Busolin ${ }^{1}$ \\ ${ }^{1}$ School of Mechanical, Aerospace and Civil Engineering, The University of Manchester, Manchester, United Kingdom.
}

\section{Key Points:}

- A physically based one-dimensional solute transport model with vertically attenuated hyporheic mixing is presented

- The behavior of the model breakthrough curves is consistent with experimental evidence

- Physical transport parameters cannot be identified solely from surface breakthrough curves unless the cross-sectional geometry is known 


\section{Abstract}

Mixing in the hyporheic zone plays a key role in controlling the fate and transport of contaminants in streams and rivers. Consistently with recent experimental and numerical results, a physically based one-dimensional solute transport model is presented that represents hyporheic mixing as a diffusion process exponentially attenuated with depth. When vertical diffusion in the sediment bed is not limited by an impermeable boundary, the moments of the BTCs generated by the model exhibit persistent non-Fickian behavior and are shown to scale consistently with existing experimental evidence. The ability of the exponentially attenuated mixing model (EAMM) to represent experimental BTCs was tested using data from field and flume tracer experiments, and its performance was compared with that of a classic two-storage zone model (TSZM). While both models provided an equally good approximation for the BTCs observed in field tracer tests, the EAMM provided better fits for the flume experiments. Analysis of the model equations and their solutions shows that, if the flow cross-sectional area and wetted perimeter are not predetermined, there are infinite sets of parameter values that produce the same space-time concentration distributions. The result implies that the physical parameters of hyporheic exchange cannot be determined by sole measurements of the solute BTCs in the surface water unless flow cross-sectional area and average flow depth can be independently estimated.

\section{Introduction}

Modeling stream-subsurface interactions is key to understanding the dispersion of waterborn contaminants in the environment. Mass and momentum exchanges between the surface water and the underlying porous bed leads contaminants into the sediment where they can be temporarily trapped or transformed through biochemical reactions. Hyporheic exchange occurs via a combination of pressure-driven Darcy flow and turbulent diffusion. The former mechanism has been widely investigated, and physically based models have been proposed to represent the advective flows and residence time distributions induced by bed forms [Elliott and Brooks, 1997; Marion et al., 2008a; Bottacin-Busolin and Marion, 2010; Chen et al., 2018], bars [Tonina and Buffington, 2007; Marzadri et al., 2010], and meanders [Boano et al., 2006; Kiel and Cardenas, 2014]. The Networks with EXchange ans Subsurface (NEXSS) model presented by Gomez-Velez and Harvey [2014] provides a network-scale mechanistic description of pressure-driven hyporheic flows induced 
by geomorphic features such as ripples, dunes, alternate bars and meanders. Turbulencedriven driven diffusion at the sediment-water interface has more recently been investigated using experimental methods [de Lemos, 2005; Higashino et al., 2009; Chandler et al., 2016; Voermans et al., 2017; Roche et al., 2019] and high-resolution numerical simulations [Breugem et al., 2006; Sherman et al., 2019].

Several formulations have been proposed to represent the effect of surface and subsurface storage processes on stream solute transport. A commonly used model is the transient storage model (TSM) [Bencala and Walters, 1983], which represents the temporary trapping of a solute in a single storage zone of finite cross-sectional area as a first-order mass transfer process. A two zone version of the transient storage model was initially developed by Choi et al. [2000] and later applied in several field tracer studies [Gooseff et al., 2004; Briggs et al., 2009; Bottacin-Busolin et al., 2011]. A one-dimensional model combining advection and dispersion in the main flow channel with Elliott and Brooks' advective pumping theory [1997] was presented by Wörman et al. [2002]. Haggerty et al. [2000] presented a general multi-rate mass transfer framework and found good agreement between experimental data from a second-order mountain stream and model breakthrough curves (BTCs) assuming a power-law residence time distribution [Haggerty et al., 2002]. Boano et al. [2007] presented an application of the Continuous Time Random Walk (CTRW) theory to solute transport in rivers and used a power-law residence time distribution to represent bed form induced hyporheic exchange. Marion et al. [2008b] proposed a general residence time formulation that decomposes transport into a residence time distribution (RTD) in the main channel, an RTD in the storage zones, and a trapping probability. Fractional dispersion approaches have also been suggested [Deng et al., 2006; Kelly et al., 2017]. Recently, Sherman et al. [2019] presented a 1-D dual domain coupled Continuous Time Random Walk (ddc-CTRW) model to represent transport in open channels with hyporheic exchange and demonstrated the potential of the model to reproduce the BTCs generated by direct numerical simulations (DNS) of turbulent transport.

A number of studies have attempted to model hyporheic exchange as a diffusive process characterized by an effective diffusion coefficient [Jackman et al., 1984; Packman et al., 2004; Marion and Zaramella, 2005; O'Connor and Harvey, 2008; Higashino et al., 2009; Grant et al., 2012]. It is well-known, however, that the dispersion coefficient in the porous medium varies with the depth from the sediment-water interface. Experimental studies [Chandler et al., 2016; Voermans et al., 2017; Roche et al., 2018] and nu- 
merical simulations [Breugem et al., 2006; Sherman et al., 2019] of hyporheic flow through flat sediment beds suggest that the mixing coefficient decreases exponentially with depth. A similar behavior is expected in the case of bed form-induced hyporheic exchange as the Darcy flow in the sediment bed is exponentially attenuated [Elliott and Brooks, 1997; Bottacin-Busolin and Marion, 2010].

Although one-dimensional transport models have often been used in combination with tracer studies to characterize stream transport processes, the physical interpretation of the model parameters has been hindered by the limited understanding of hyporheic mixing and its effect on in-stream solute transport [Marion et al., 2003; Ward et al., 2013; Wondzell, 2006; Zaramella et al., 2016]. A number of studies have pointed out that the parameters of classic 1-D transport models depend on the length of the study reach [BottacinBusolin et al., 2011; Gooseff et al., 2013], and clear experimental evidence has been provided that the spatio-temporal evolution of the BTCs in stream tracer studies is inconsistent with the predictions of classic 1-D transport models [Nordin and Troutman, 1980; González-Pinzón et al., 2013]. Recently, Bottacin-Busolin [2017] used particle-tracking simulations to show that the anomalous scaling of the moments of the BTCs can be explained by the vertical variation of the mixing coefficient in the porous bed. A more recent study by Roche et al. [2019] also pointed out that, in addition to the effect of vertical hyporheic mixing, the streamwise velocity in the hyporheic zone can significantly affect the tail behavior of the BTCs.

Whilst existing models of hyporheic exchange focus on the advective flows induced by geomorphic features such as bed forms, bars and meanders [e.g., Elliott and Brooks, 1997; Marzadri et al., 2010; Gomez-Velez and Harvey, 2014], modeling studies by BottacinBusolin and Marion [2010] and Bottacin-Busolin [2017] have shown that the vertical variation of the dispersion coefficient in the porous medium plays a key role in determining the spatio-temporal behavior of solute breakthrough curves. Mechanistic models that neglect dispersive fluxes across the sediment-water interface are also incapable of describing transport over flat sediment beds, for which hyporheic exchange is primarily determined by laminarization and penetration of turbulence into the porous medium. Recognizing the need for an upscaled physically based 1-D model capable of representing surface and subsurface mixing, this work presents a 1-D solute transport model that takes into account the exponential attenuation of the mixing coefficient in the sediment bed. The ability of the model to represent BTCs from field and flume tracer experiments and the evolution of 
their temporal moments as a function of reach length is compared with that of a classic two-storage zone solute transport model. In addition, the analysis of the model equations and their solutions provides novel insights into the equifinality of surface and subsurface transport parameters, with significant implications for stream tracer studies.

\section{Model}

Under the assumption of complete cross-sectional mixing, the transport of a solute in an open-channel flow over a porous bed can be described as a 1-D advection-dispersion process with mass transfer at the sediment-water interface. For steady flow, the equation governing the concentration of a non-reactive solute in the main flow channel (MFC) can be written as:

$$
\frac{\partial C(x, t)}{\partial t}+U \frac{\partial C(x, t)}{\partial x}-D \frac{\partial^{2} C(x, t)}{\partial x^{2}}=-\frac{J(x, t)}{R_{H}}
$$

where $x$ is the streamwise coordinate $(\mathrm{L}), t$ is time $(\mathrm{T}), C(x, t)$ is the solute concentration in the water column $\left(\mathrm{ML}^{-3}\right), U=Q / A$ is the average flow velocity $\left(\mathrm{L} \mathrm{T}^{-1}\right), Q$ is the flow discharge $\left(\mathrm{L}^{3} \mathrm{~T}^{-1}\right), A$ is the flow cross-sectional area $\left(\mathrm{L}^{2}\right), R_{H}$ is the hydraulic radius $(\mathrm{L})$, $D$ is the longitudinal dispersion coefficient $\left(\mathrm{L}^{2} \mathrm{~T}^{-1}\right)$, and $J$ is the interfacial mass flux at the sediment-water interface $\left(\mathrm{ML}^{-2} \mathrm{~T}^{-1}\right)$. More generally, the flux $J$ can be used to describe exchange processes at the interface between the MFC and slow mixing zones such as side pockets or vegetated zones. Note that equation (1) assumes that $J$ is the average mass flux along the wetted perimeter. In applications where the lateral exchange flux is negligible, $R_{H}$ should be replaced by the flow depth, $h(\mathrm{~L})$.

Recent studies have shown that mixing in the hyporheic zone can be described as a diffusion process with a mixing coefficient exponentially attenuated with depth. In the case of turbulent flow over a flat sediment bed, hyporheic exchange is primarily diffusive in nature [Chandler et al., 2016]. In the case of flow over bed forms, the seepage velocity induced by the pressure variation on the bed surface is known to be exponentially attenuated with depth [Elliott and Brooks, 1997; Bottacin-Busolin and Marion, 2010], and therefore a similar behavior is expected for the dispersion coefficient in the porous medium. As pointed out by Bottacin-Busolin [2017], if the velocity in the porous medium is much smaller than the average velocity in the main channel, the vertical velocity distribution in the porous medium has negligible effect on the longitudinal dispersion process, which is instead dominated by the vertical variation of the mixing coefficient. 
For exponentially damped diffusion in the vertical direction of the porous medium, the mass flux at the sediment-water interface can be expressed as:

$$
J(x, t)=\left.\varepsilon_{s} \frac{\partial C_{s}(x, z, t)}{\partial z}\right|_{z=0}
$$

where $z$ is the vertical coordinate $(\mathrm{L})$, positive upward and with $z=0$ at the sedimentwater interface, $C_{s}(x, z, t)$ is the concentration in the sediment $\left(\mathrm{ML}^{-3}\right)$, and $\varepsilon_{s}$ is the interfacial mixing coefficient $\left(\mathrm{L}^{2} \mathrm{~T}^{-1}\right)$. In the sediment bed, the concentration $C_{s}$ satisfies the equation:

$$
\frac{\partial C_{s}}{\partial t}+u_{s} \frac{\partial C_{s}}{\partial x}-\frac{\partial}{\partial z}\left(\varepsilon(z) \frac{\partial C_{s}}{\partial z}\right)=0
$$

where $u_{s}$ is the average velocity of the subsurface flow induced by the stream gradient, and $\varepsilon(z)$ is the vertical mixing coefficient, such that $\varepsilon=\varepsilon_{s}$ at $z=0$. At the sediment-water interface the subsurface concentration is assumed to be the same as the concentration in the water column,

$$
C_{s}(x, z=0, t)=C(x, t)
$$

whereas, for a bed of finite depth $d_{s}(\mathrm{~L})$, a no-flux condition is assumed at the bottom boundary,

$$
\left.\frac{\partial C_{s}(x, z, t)}{\partial z}\right|_{z=-d_{s}}=0
$$

The mixing coefficient $\varepsilon(z)$ can be used to represent dispersive fluxes induced by the laminarization of turbulent eddies at the sediment-water interface and the penetration of turbulence into the porous medium. Turbulent momentum transfer across the stream-subsurface interface has been shown to produce non-Darcy flows that decrease exponentially with depth in the streambed [Zhou and Mendoza, 1993; Packman et al., 2004; Breugem et al., 2006]. For flat sediment beds, Chandler et al. [2016] experimentally investigated the vertical variation of the in-bed mixing coefficient and proposed an empirical relationship linking the mixing coefficient to the the sediment permeability and bed shear velocity. Mixing in the hyporheic zone is further enhanced by the interaction between the stream turbulent flow and surface bed forms such as ripples and dunes, which induce Darcy flows in the sediment bed that are exponentially attenuated with depth [Elliott and Brooks, 1997]. Dispersion in the porous medium and the vertical variation of the dispersion tensor have been shown to significantly affect the residence time distributions of bed form-induced hyporheic exchange [Bottacin-Busolin and Marion, 2010]. Following Bottacin-Busolin [2017] [see also Roche et al., 2019], the mixing coefficient $\varepsilon(z)$ in the porous bed is expressed as 
follows:

$$
\varepsilon(z)=\varepsilon_{\infty}+\left(\varepsilon_{s}-\varepsilon_{\infty}\right) e^{z / h_{s}}
$$

where $h_{s}$ is the attenuation depth (L) and $\varepsilon_{\infty}$ is the asymptotic mixing coefficient in the sediment ( $\left(\mathrm{L}^{2} \mathrm{~T}^{-1}\right)$ such that $\varepsilon=\varepsilon_{\infty}$ at $z=-\infty$. Equations (1), (2) and (3) with the boundary conditions (4)-(5) can be solved in the Laplace domain as described in Appendix A.

An alternative parametrization assumes that the exchange with the storage zone is proportional to the difference between the concentration in the surface water and the concentration in storage zones of finite cross-sectional areas, $A_{s, i}$ :

$$
J(x, t)=R_{H} \sum_{i=1}^{N} \alpha_{i}\left[C(x, t)-C_{s, i}(x, t)\right]
$$

where $N$ is the number of storage domains. The concentration in the $i$-th storage domain satisfies the equation

$$
\frac{\partial C_{s, i}}{\partial t}=\frac{C-C_{s, i}}{T_{i}}
$$

where $T_{i}=A_{s, i} /\left(A \alpha_{i}\right)$ is the average detention time in the $i$-th storage zone (T). This formulation represents a multi-storage zone extension of the Transient Storage Model (TSM) [Choi et al., 2000]. Several field tracer studies have shown that a two-storage zone model (TSZM) with exchange flux expressed by (7) with $N=2$, generally provides a good approximation for the observed tracer BTCs [Gooseff et al., 2004; Briggs et al., 2009; Bottacin-Busolin et al., 2011; Zaramella et al., 2016]. The two-storage zone model is often justified by associating one of the storage components with surface storage, and the second component with hyporheic exchange.

\section{Numerical Tests}

In order to analyze the behavior of the concentration distributions generated by the model, it is convenient to reduce the number of parameters by nondimensionalizing the model equations. Equation (1) is nondimensionalized as follows:

$$
\frac{\partial \hat{C}(\hat{x}, \hat{t})}{\partial \hat{t}}+\frac{\partial \hat{C}(\hat{x}, \hat{t})}{\partial \hat{x}}-\hat{D} \frac{\partial^{2} \hat{C}(\hat{x}, \hat{t})}{\partial \hat{x}^{2}}=-\hat{J}(\hat{x}, \hat{t})
$$

where $\hat{x}=x / R_{H}$ is the nondimensional distance, $\hat{t}=U t / R_{H}$ is the nondimensional time, $\hat{C}=C / C_{r}$ is the normalized concentration relative to the reference concentration $C_{r}, \hat{D}=$ $D /\left(U R_{H}\right)$ is the normalized longitudinal dispersion coefficient, $\hat{J}=J /\left(C_{r} U\right)$ is the normalized exchange flux. For the EAMM, the interfacial mass flux is

$$
\hat{J}(\hat{x}, \hat{t})=\left.\hat{\varepsilon}_{s} \frac{\partial \hat{C}_{s}(\hat{x}, \hat{z}, \hat{t})}{\partial \hat{z}}\right|_{\hat{z}=0}
$$


where the normalized concentration in the sediment $\hat{C}_{s}=C_{s} / C_{r}$ satisfies the equation

$$
\frac{\partial \hat{C}_{s}}{\partial \hat{t}}+\hat{u}_{s} \frac{\partial \hat{C}_{s}}{\partial \hat{x}}-\frac{\partial}{\partial \hat{z}}\left(\hat{\varepsilon}(\hat{z}) \frac{\partial \hat{C}_{s}}{\partial \hat{z}}\right)=0
$$

where $\hat{z}=z / R_{H}, \hat{u}_{s}=u_{s} / U$ and $\hat{\varepsilon}_{s}=\varepsilon_{s} /\left(U R_{H}\right)$. Accordingly, the nondimensional attenuation depth is defined as $\hat{h}_{s}=h_{s} / R_{H}$ and the nondimensional sediment depth is $\hat{d}_{s}=$ $d_{s} / R_{H}$. Here, $R_{H}$ is used as the scaling length consistently with equation (1) where $J$ is defined as the average mass flux along the wetted perimeter. However, if only vertical exchange is considered, $R_{H}$ should be replaced by the flow depth, $h$. For the TSZM, the interfacial mass flux and storage equations are, respectively:

$$
\begin{aligned}
\hat{J}(\hat{x}, \hat{t}) & =\sum_{i=1}^{2} \hat{\alpha}_{i}\left[\hat{C}(\hat{x}, \hat{t})-\hat{C}_{s, i}(\hat{x}, \hat{t})\right] \\
\frac{\partial \hat{C}_{s, i}}{\partial \hat{t}} & =\frac{\hat{C}-\hat{C}_{s, i}}{\hat{T}_{i}} \quad i=1,2
\end{aligned}
$$

where $\hat{\alpha}_{i}=\alpha_{i} R_{H} / U$ and $\hat{T}_{i}=U T_{i} / R_{H}$.

The BTCs generated by the EAMM for the parameters in Table 1 are presented in Figure 1. The figure shows that the EAMM can generate breakthrough curves with three main types of tail behaviors: 1) when the subsurface flow velocity is non-zero (Runs 1 and 2), i.e., $u_{s}>0$, the tails exhibit rapid tempering as the time approaches the maximum travel time $L / u_{s}$, where $L$ is the reach length;2) if the subsurface flow velocity is zero, the sediment depth is finite, and the asymptotic diffusivity $\varepsilon_{\infty}>0$ (Runs 5, 6,8,9 and 10), then the tails of the BTCs follow an exponential law; 3) when the subsurface flow velocity is zero and the sediment depth is infinite (Runs 3,4 and 7), the tails of the BTCs exhibit power-law behavior. In the latter case, we can distinguish between two possible asymptotic behaviors: i) if the asymptotic diffusivity $\varepsilon_{\infty}>0, C \sim t^{-3 / 2}$ as $t \rightarrow \infty$; ii) if $\varepsilon_{\infty}=0$, the numerically generated BTCs suggest $C \sim t^{-2}$ as $t \rightarrow \infty$. Intermediate powerlaw exponents between $-3 / 2$ and -2 can be observed within finite time intervals for relatively small values of $\varepsilon_{\infty}$. Power-law tail behavior can also be observed when the sediment depth is finite, provided that $d_{s} \gg h_{s}$ and $d_{s}^{2} / \varepsilon_{\infty} \gg x / u$. Note that the scaling exponent -2 resulting from an exponentially damped diffusion process is also consistent with the late-time behavior of the residence time distributions in the sediment for bed forminduced advective pumping [Boano et al., 2007; Marion et al., 2008b; Bottacin-Busolin and Marion, 2010].

The ability of a transport model to fit a breakthrough curve at a section does not imply the ability to represent the space-time behavior of the concentration distributions. 
Table 1. Parameters of the EAMM used to generate the breakthrough curves in Figure 1.

\begin{tabular}{lrrrrrrrrrr}
\hline Run & 1 & 2 & 3 & 4 & 5 & 6 & 7 & 8 & 9 & 10 \\
\hline$\hat{D}$ & 100 & 100 & 100 & 100 & 100 & 100 & 100 & 100 & 100 & 100 \\
$\hat{\varepsilon}_{s}\left(\times 10^{-3}\right)$ & 1.0 & 1.0 & 1.0 & 1.0 & 1.0 & 0.01 & 0.001 & 0.01 & 1.0 & 1.0 \\
$\hat{\varepsilon}_{\infty}\left(\times 10^{-3}\right)$ & 0.01 & 0.01 & 0.01 & 0 & 0.01 & 0.01 & 0 & 0.01 & 1.0 & 1.0 \\
$\hat{h}_{s}$ & 0.02 & 0.02 & 0.02 & 0.02 & 0.02 & 0.02 & 0.02 & 0.02 & 0.02 & 0.02 \\
$\hat{d}_{s}$ & $\infty$ & $\infty$ & $\infty$ & $\infty$ & 0.3 & 0.5 & $\infty$ & 0.3 & 1.0 & 2.0 \\
$\hat{u}_{s}$ & 0.2 & 0.1 & 0 & 0 & 0 & 0 & 0 & 0 & 0 & 0 \\
\hline
\end{tabular}
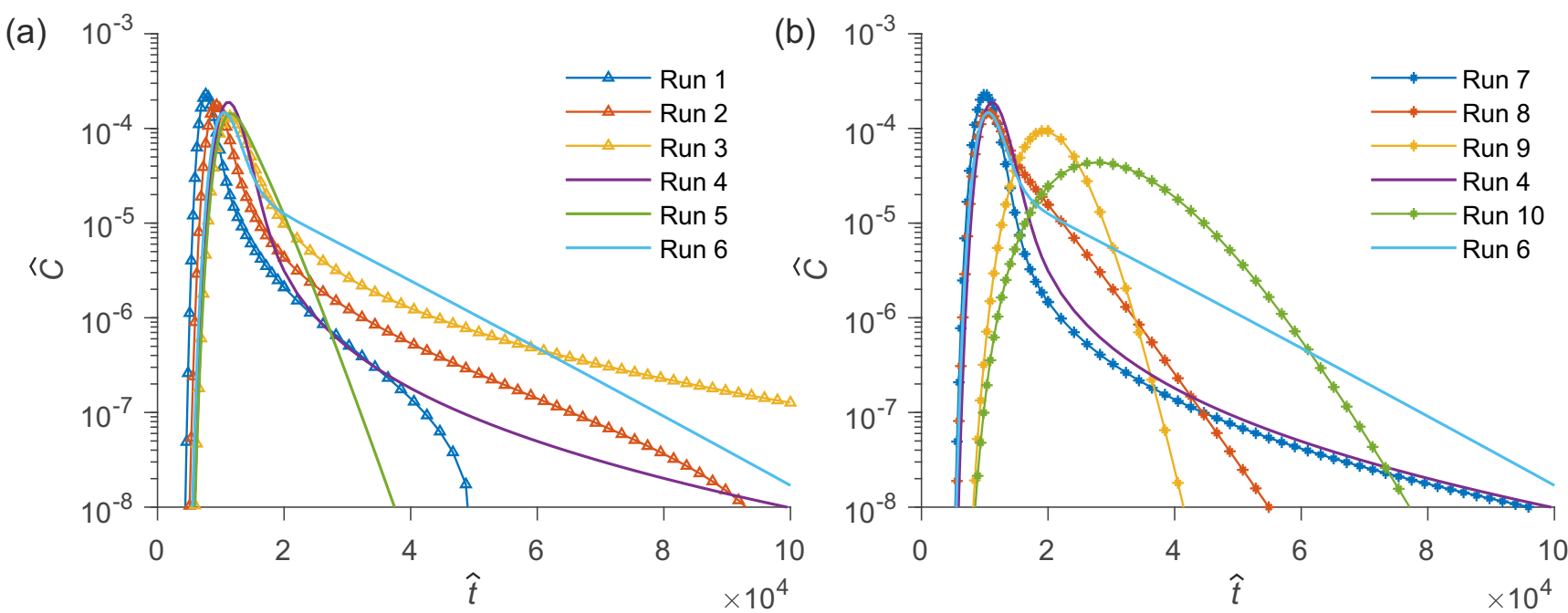

Figure 1. Breakthrough curves generated by the EAMM for the parameters in Table 1. The markers are only used to better differentiate the curves.

Experimental evidence from field tracer studies has shown that the scaling of the moments of tracer breakthrough curves with the distance from the injection point is inconsistent with classic solute transport models [González-Pinzón et al., 2013]. The observed non-Fickian scaling of the moments of the BTCs and the persistence of the skewness at late times has been shown to be consistent with particle-tracking simulations of transport in open-channel flow with vertical attenuation of the mixing coefficient in the porous bed [Bottacin-Busolin, 2017]. A comparison between the first nondimensional moment, $\hat{m}_{t, 1}$, variance, $\hat{m}_{t, 2}$, and coefficient of skewness (CSK), $\gamma_{t}$, of the BTCs generated by the EAMM and TSZM with the parameters in Table 2 for a pulse injection is presented in Figure 2 as a function of the distance from the injection point. The moments were ob- 
Table 2. Parameters of the EAMM and TSZM considered in the moment analysis.

\begin{tabular}{|c|c|c|c|c|c|c|c|c|c|c|}
\hline \multicolumn{6}{|c|}{ (a) EAMM } & \multicolumn{5}{|c|}{ (b) TSZM } \\
\hline Run & $\hat{\varepsilon}_{s}$ & $\hat{h}_{s}$ & $\hat{d}_{s}$ & $\hat{\varepsilon}_{\infty}$ & $\hat{u}_{s}$ & Run & $\hat{\alpha}_{1}$ & $\hat{T}_{1}$ & $\hat{\alpha}_{2}$ & $\hat{T}_{2}$ \\
\hline A-1 & $10^{-4}$ & 0.02 & $\infty$ & 0 & 0 & B-1 & 0 & 20 & 0 & \\
\hline A-2 & $10^{-4}$ & 0.1 & $\infty$ & 0 & 0 & B-2 & 0.01 & 100 & 0 & \\
\hline A-3 & 0.1 & 0.02 & $\infty$ & 0 & 0 & B-3 & $10^{-3}$ & 100 & $10^{-5}$ & 1000 \\
\hline A-4 & 0.1 & 0.1 & $\infty$ & 0 & 0 & B-4 & $10^{-3}$ & 100 & $10^{-5}$ & 3000 \\
\hline A-5 & $10^{-3}$ & 0.05 & 0.3 & 0 & 0 & B-5 & $10^{-3}$ & 100 & $10^{-5}$ & 5000 \\
\hline A-6 & $10^{-3}$ & 0.05 & 0.1 & 0 & 0 & B-6 & $10^{-3}$ & 100 & $3 \times 10^{-5}$ & 5000 \\
\hline A-7 & $10^{-4}$ & 0.05 & $\infty$ & $10^{-4}$ & 0 & B-7 & $3 \times 10^{-3}$ & 100 & $6 \times 10^{-5}$ & 5000 \\
\hline
\end{tabular}

tained numerically from the simulated BTCs truncated at $1 \%$ of the peak concentration value. It must be stressed that when the tails of the BTCs scale asymptotically as $t^{\kappa}$ with $\kappa \geq-2$, the moments are not finite, and therefore the concentration moments significantly depend on the BTC truncation threshold. This should be taken into account when comparing model results with experimental observations [Drummond et al., 2012]. It should also be noted that, although in reality the moments are always finite due to the maximum residence time imposed by the non-zero subsurface velocity $u_{s}$ [Roche et al., 2019], the rapid tempering of the tail associated with the subsurface flow may only be observable in practice if the substrate material has a relatively high hydraulic conductivity.

The first moment of the BTCs generated by both models is asymptotically linear except for the EAMM with $\varepsilon_{s}=\varepsilon_{\infty}>0$ (Run A-7). In all simulations with the TSZM, the variance is asymptotically linear, whereas the EAMM has asymptotically linear variance only in the case of a finite sediment depth (Runs A-5 and A-6). For beds with infinite depth and $\varepsilon_{\infty}=0$, the variance of the BTCs produced by the EAMM has power-law exponents between 1.5 and 2 within the simulation interval, and therefore the longitudinal dispersion process is superdiffusive. For Run A-7, where $\varepsilon_{s}=\varepsilon_{\infty}>0$, the variance has power-law exponent greater than 2 . When the porous medium is assumed to have finite thickness, the EAMM produces solute BTCs that have similar behavior to those produced by a first-order mass transfer model.

The behavior of the CSK is shown in Figure 2c for the EAMM, and in Figure $2 \mathrm{f}$ for TSZM. In all the runs conducted with the TSZM, the CSK decreases with $t^{-1 / 2}$ at large 

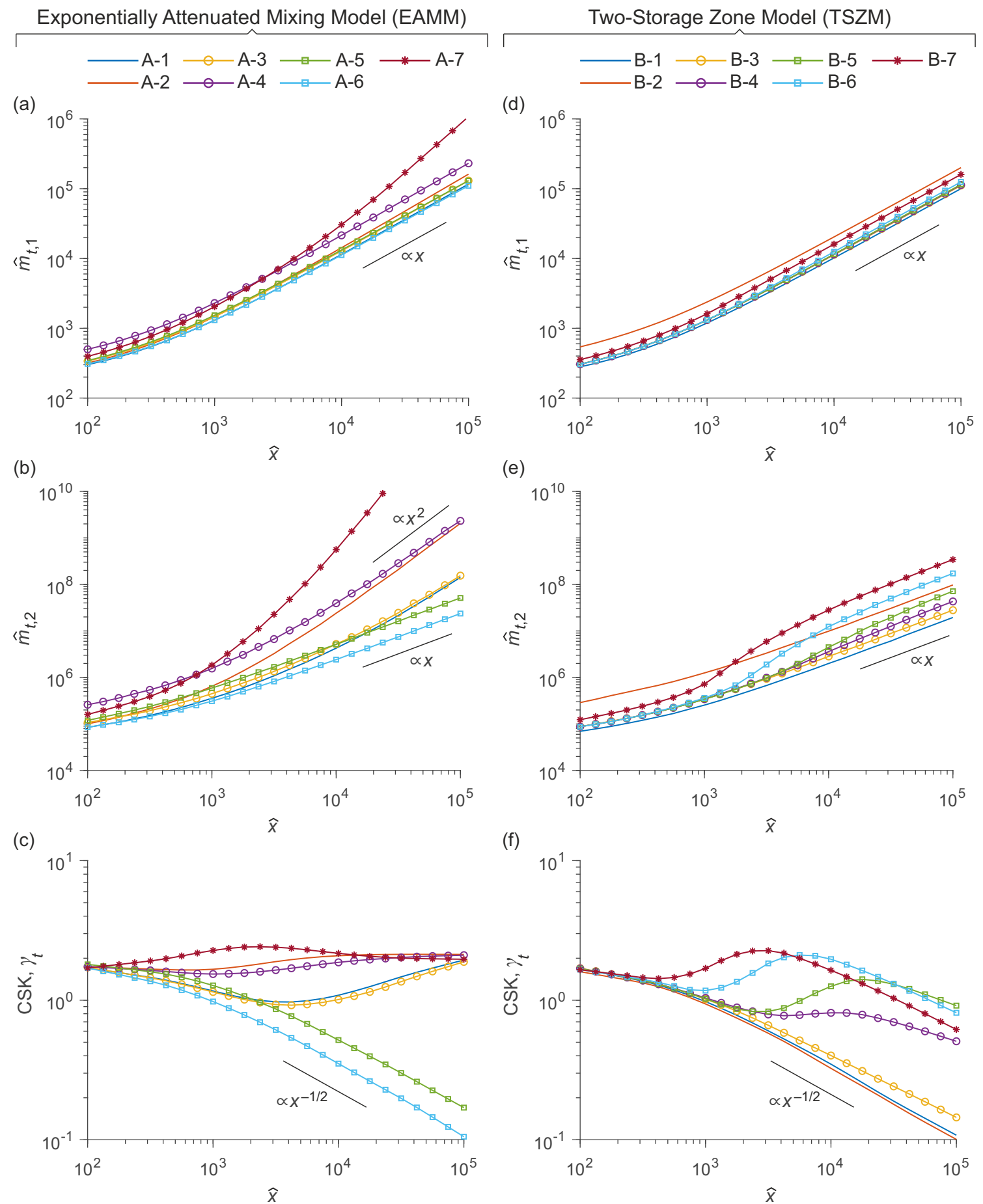

Figure 2. Moments of the truncated BTCs generated by EAMM and TSZM for the parameters in Table 2 as a function of the distance from the injection point. The BTCs were truncated at $1 \%$ of the peak concentration value.

times consistently with a Fickian diffusion process, whereas in the case of the EAMM with infinite sediment depth, the CSK has a non-decreasing behavior over a wide range of 
timescales. Only the runs conducted with a bed of finite thickness have a CSK that decays as $t^{-1 / 2}$. The superdiffusive scaling of the variance and the persistence of the skewness of the BTCs generated by the EAMM is consistent with experimental evidence reported in the literature [Day, 1975; Nordin and Troutman, 1980; González-Pinzón et al., 2013].

\section{Comparison with Experimental Data}

\subsection{Field Tracer Tests}

The ability of the model to represent data from field tracer studies was tested using a best-fit approach. Although the EAMM was conceived to represent hyporheic mixing in line with experimental evidence indicating an exponential attenuation of the mixing coefficient in the streambed, the model parameters determined by inverse modeling of field tracer data are expected to represent the combined effect of surface storage and hyporheic exchange. The comparison between best-fit model BTCs and the experimental observations presented here is therefore not intended to validate the model, but to provide evidence of the ability of the model to reproduce the shape and tail behavior of the experimental BTCs.

The tracer data used in this study were taken from the work of Bottacin-Busolin et al. [2011], and refer to experiments conducted in the Desturo Canal, Italy, and the Yarqon River, Israel. The BTC from the Desturo Canal refers to a plateau injection of rhodamine WT in a $262 \mathrm{~m}$ long reach, whereas the data from the Yarqon River refers to a plateau injection in a $1887 \mathrm{~m}$ long reach. In both cases the tracer was injected at the start of the experimental reach, and the concentration was measured at the end. The river discharge and the flow cross-sectional area were determined from experimental data as described by Bottacin-Busolin et al. [2011]. To avoid overparameterization, it is assumed that $\varepsilon_{\infty}=0$. Also, given the small bed slope and the low hydraulic conductivity of the sediment, the underflow velocity induced by the stream gradient is expected to be negligible relative to the average velocity in the stream channel, hence $u_{s}=0$. The underlying assumption is that the longitudinal distance traveled by the tracer in the sediment is significantly smaller than the distance traveled in the MFC, and therefore the effect of the underflow velocity $u_{s}$ on the surface BTCs within the time frame of observation is expected to be negligible. Accordingly, the calibration parameters are $D, \varepsilon_{s}, h_{s}$ and $d_{s}$ for the EAMM, and $D, \alpha_{1}$, $\alpha_{2}, T_{1}$ and $T_{2}$ for the TSZM. These were determined by best-fitting the experimental data 
relative to a mixed-scale root mean square error (RMSE) calculated as in Bottacin-Busolin et al. [2011]:

$$
\mathrm{RMSE}=\sqrt{\frac{1}{N_{\mathrm{obs}}}\left(\sum_{j \in I_{U}} \frac{\left(C_{j}-C_{\mathrm{obs}, j}\right)^{2}}{\left(C_{\mathrm{obs}, \text { max }}-C_{\mathrm{obs}, \min }\right)^{2}}+\sum_{j \in I_{L}} \frac{\left(\log C_{j}-\log C_{\mathrm{obs}, j}\right)^{2}}{\left(\log C_{\mathrm{obs}, \max }-\log C_{\mathrm{obs}, \min }\right)^{2}}\right)}
$$

where $N_{\mathrm{obs}}$ is the number of observations, $C_{j}$ and $C_{\mathrm{obs}, j}$ are respectively the simulated and observed tracer concentrations, $C_{\mathrm{obs}, \min }$ and $C_{\mathrm{obs}, \max }$ are respectively the observed minimum and maximum concentrations, and $I_{U}$ and $I_{L}$ are the sets of observed values respectively higher and lower than $20 \%$ of the maximum concentration. In applying equation (14), concentrations lower than 5\% of the peak concentration were excluded. The minimization of the RMSE was carried out using the Differential Evolution algorithm for global optimization of Storn and Price [1997].

The best-fit model parameters are presented in Table 3 and the resulting BTCs are shown in Figure 3. The figure shows that both models well approximate the observed concentrations. The TSZM provides a slightly better fit for the Desturo Canal compared to the EAMM, whereas the EAMM slightly outperforms the TSZM for the Yarqon River. In the case of the Desturo Canal, both models generate BTCs with exponentially decreasing tail concentrations. For the EAMM, this implies that the best-fit sediment depth $d_{s} / R_{H}$ has a finite value. In the case of the Yarqon river, instead, the best-fit sediment depth is sufficiently large to make the model insensitive to this parameter, so that $d_{s}$ can be taken as $\infty$.

In assessing the relative performance of the two models, it should be noted that here the EAMM has only 4 calibration parameters as opposed to 5 in the TSZM. Also, due to the properties of the substrate material in the Desturo Canal [Bottacin-Busolin et al., 2011] and the relatively short study reach, the effect of hyporheic exchange on the BTCs is expected to be small, therefore the deviation from the classic dispersion theory is likely to be associated primarily with surface transient storage.

\subsection{Flume Tracer Tests}

The ability of the model to represent experimental BTCs was also tested against data from flume tracer experiments. The data used here was taken from the work of BottacinBusolin et al. [2009], who investigated the role of bed morphology and biofilm growth on the transient storage of solutes. In these experiments, the flume was $40 \mathrm{~m}$ long and $40 \mathrm{~cm}$ wide with the bottom shaped as impermeable bed forms of $1 \mathrm{~m}$ length. The height of the 
Table 3. Calibrated model parameters for field tracer experiments.

\begin{tabular}{|c|c|c|c|c|c|c|c|c|c|c|c|}
\hline \multirow[b]{2}{*}{ Test } & \multirow[b]{2}{*}{$\begin{array}{c}Q \\
\left(\mathrm{~m}^{3} \mathrm{~s}^{-1}\right)\end{array}$} & \multirow[b]{2}{*}{$\begin{array}{c}A \\
\left(\mathrm{~m}^{2}\right)\end{array}$} & \multirow[b]{2}{*}{$\begin{array}{c}D \\
\left(\mathrm{~m}^{2} \mathrm{~s}^{-1}\right)\end{array}$} & \multicolumn{4}{|c|}{ EAMM } & \multicolumn{4}{|c|}{ TSZM } \\
\hline & & & & $\begin{array}{c}\varepsilon_{S} / R_{H}^{2} \\
\left(\times 10^{-3} \mathrm{~s}^{-1}\right)\end{array}$ & $\begin{array}{c}h_{S} / R_{H} \\
(-)\end{array}$ & $\begin{array}{c}d_{S} / R_{H} \\
\quad(-)\end{array}$ & $\begin{array}{c}u_{s} \\
\left(\mathrm{~m} \mathrm{~s}^{-1}\right)\end{array}$ & $\begin{array}{c}\alpha_{1} \\
\left(\times 10^{-4} \mathrm{~s}^{-1}\right)\end{array}$ & $\begin{array}{l}T_{1} \\
\text { (s) }\end{array}$ & $\begin{array}{c}\alpha_{2} \\
\left(\times 10^{-4} \mathrm{~s}^{-1}\right)\end{array}$ & $\begin{array}{l}T_{2} \\
(\mathrm{~s})\end{array}$ \\
\hline \multirow[t]{2}{*}{ Desturo } & 0.045 & 0.19 & 0.20 & 0.344 & 0.0624 & 0.249 & 0 & & & & \\
\hline & 0.045 & 0.19 & 0.10 & & & & & 12.50 & 99 & 2.730 & 420 \\
\hline \multirow[t]{2}{*}{ Yarqon } & 0.410 & 2.15 & 2.99 & 0.156 & 0.1279 & $\infty$ & 0 & & & & \\
\hline & 0.410 & 2.15 & 2.17 & & & & & 5.02 & 525 & 0.485 & 5103 \\
\hline
\end{tabular}
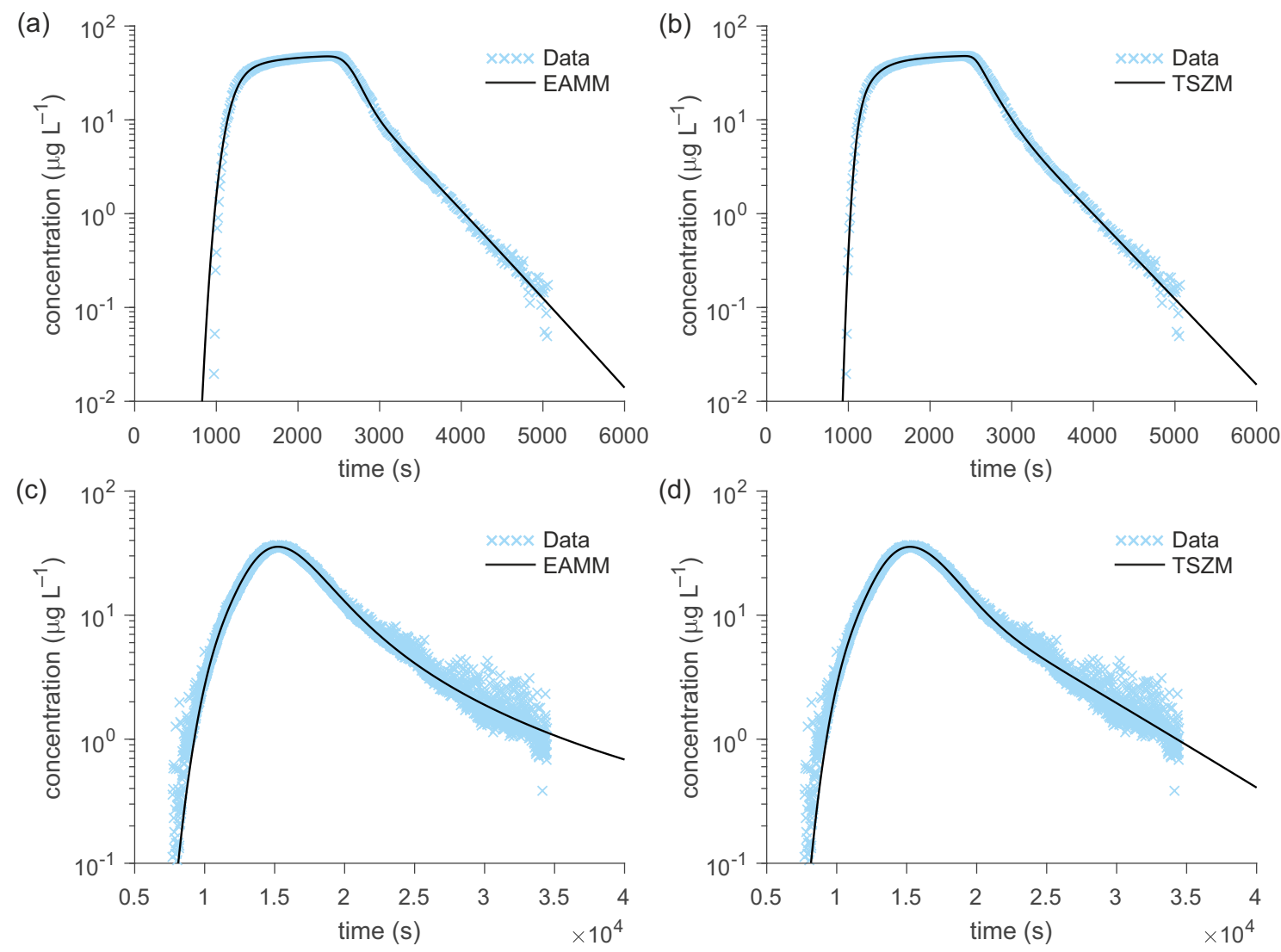

Figure 3. Experimental data from Bottacin-Busolin et al.'s field tracer experiments [2011] and best-fit model BTCs for (a-b) Desturo Canal and (c-d) Yarqon River.

bed form was $4 \mathrm{~cm}$ in test PB15F04 and $10 \mathrm{~cm}$ in test PB21F10. The impermeable bed forms were covered with a single layer of graded sediment with mean diameter $9.2 \mathrm{~mm}$ as substratum for biofilm growth [Bottacin-Busolin et al., 2009]. At the time of the experiments, biofilm had been growing for 15 days in test PB15F04 and 21 days in test 
Table 4. Calibrated model parameters for flume experiments.

PB21F10. In the tests, a plateau injection of rhodamine WT was made at the upstream end of the flume, and the tracer concentration was measured at the downstream end. The flume was continuously fed with raw stream water in a flow-through mode. Following a similar approach to that described in section 4.1 , it was assumed that $\varepsilon_{\infty}=0$, but $u_{s}$ was kept as a calibration parameter in view of the coarse size of the bed sediment.

The best-fit model parameters are presented in Table 4 and the resulting BTCs are shown in Figure 4. Notice that the hydraulic radius, $R_{H}$, is here replaced by the flow depth, $h$, because the impermeable walls and the geometry of the experiment did not allow any significant lateral exchange, and therefore the parameters $\varepsilon_{s}, h_{s}$ and $d_{s}$ are assumed to represent vertical exchange only. It can be seen that the long-tail behavior of the experimental BTCs is well represented by the EAMM but not the TSZM. In particular, in experiment PB15F04 the plateau phase of the BTC generated by the TSZM is followed by three main slopes in a semi-log scale: the first slope is associated with surface dispersion, whereas the second and third slopes are associated with fast and slow transient storage, respectively. These three slopes are clearly recognizable in the BTC generated by the TSZM, whilst the continuous change in slope in the experimental data is consistent with the power-law behavior of the BTC produced by the EAMM.

\begin{tabular}{|c|c|c|c|c|c|c|c|c|c|c|c|}
\hline \multirow[b]{2}{*}{ Test } & \multirow[b]{2}{*}{$\begin{array}{c}Q \\
\left(\mathrm{~L} \mathrm{~s}^{-1}\right)\end{array}$} & \multirow[b]{2}{*}{$\begin{array}{c}A \\
\left(\mathrm{~m}^{2}\right)\end{array}$} & \multirow[b]{2}{*}{$\begin{array}{c}D \\
\left(\mathrm{~m}^{2} \mathrm{~s}^{-1}\right)\end{array}$} & \multicolumn{4}{|c|}{ EAMM } & \multicolumn{4}{|c|}{ TSZM } \\
\hline & & & & $\begin{array}{c}\varepsilon_{S} / h^{2} \\
\left(\times 10^{-3} \mathrm{~s}^{-1}\right)\end{array}$ & $\begin{array}{c}h_{S} / h \\
(-)\end{array}$ & $\begin{array}{c}d_{S} / h \\
(-)\end{array}$ & $\begin{array}{c}u_{s} \\
\left(\mathrm{~m} \mathrm{~s}^{-1}\right)\end{array}$ & $\begin{array}{c}\alpha_{1} \\
\left(\times 10^{-4} \mathrm{~s}^{-1}\right)\end{array}$ & $\begin{array}{l}T_{1} \\
\text { (s) }\end{array}$ & $\begin{array}{c}\alpha_{2} \\
\left(\times 10^{-4} \mathrm{~s}^{-1}\right)\end{array}$ & $\begin{array}{l}T_{2} \\
(\mathrm{~s})\end{array}$ \\
\hline \multirow[t]{2}{*}{ PB15F04 } & 2.25 & 0.0279 & 0.014 & 0.112 & 0.0312 & 0.262 & 0.0021 & & & & \\
\hline & 2.25 & 0.0279 & 0.025 & & & & & 1.00 & 427 & 0.158 & 2340 \\
\hline \multirow[t]{2}{*}{ PB21F10 } & 2.25 & 0.0390 & 0.016 & 6.41 & 0.0319 & 0.370 & 0.0034 & & & & \\
\hline & 2.25 & 0.0390 & 0.020 & & & & & 2.03 & 161 & 0.548 & 846 \\
\hline
\end{tabular}

\section{Equifinality of Model Parameters}

Tracer studies are often used in conjunction with one-dimensional solute transport models to characterize stream transport processes. However, the possibility of representing the same breakthrough curves using different sets of parameter values can lead to uncertainties in the physical interpretation of the model parameters [Bottacin-Busolin et al., 

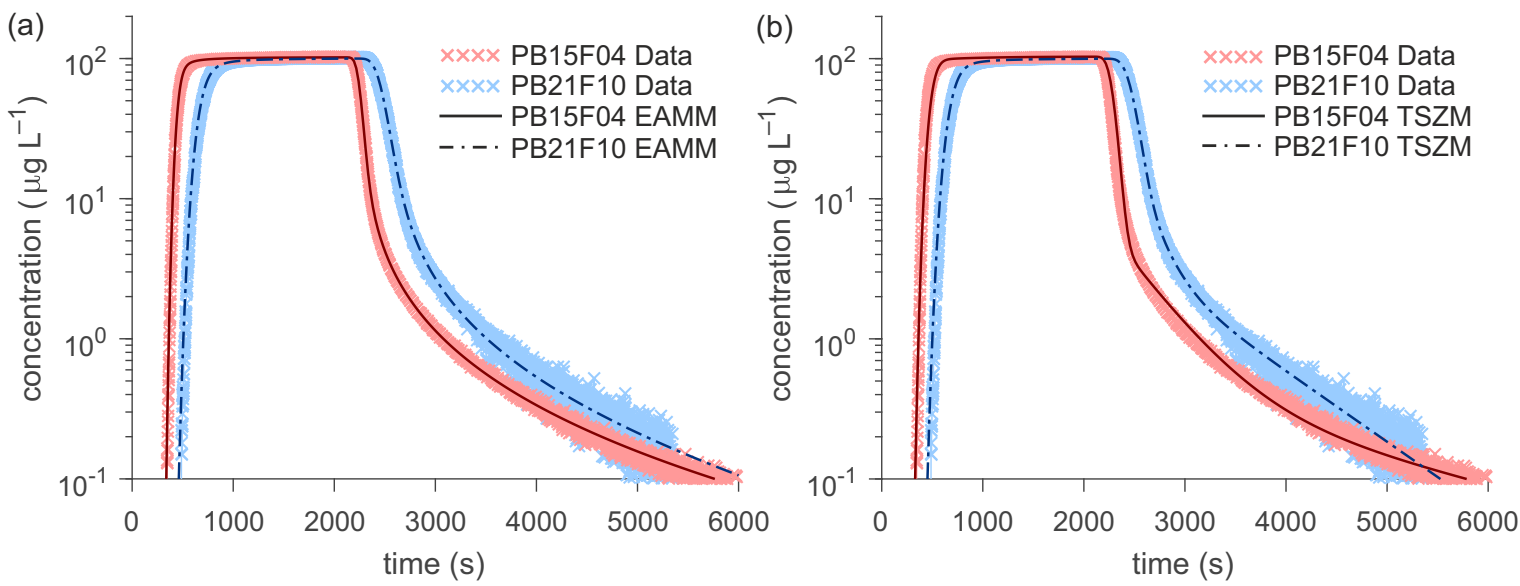

Figure 4. Experimental data from Bottacin-Busolin et al.'s flume experiments [2009] and best-fit model BTCs.

2011; Gooseff et al., 2013; Zaramella et al., 2016]. This problem is commonly referred to as equifinality or non-uniqueness in parameter estimation [Beven, 2006]. A number of studies have investigated the identifiability of the TSM parameters. Kelleher et al. [2013] applied the TSM to pulse tracer data from a mountain stream and found that, depending on stream setting, only some of the processes represented in the TSM were identifiable. Rana et al. [2019] analyzed the uncertainty in the TSM parameters for different stream flow magnitudes and found that the storage parameters of the TSM are less significant for higher flows. Kelleher et al. [2019] investigated whether observations from different tracers can lead to reduced uncertainty, and concluded that nonconservative tracers can help improve parameter interpretation. Variations in stream geometry also contribute to uncertainty [Schmadel et al., 2014].

The analysis of the EAMM equations shows that there are two sources of equifinality in the model. First, inspection of equations (1), (2) and (3) shows that, if only the concentration $C(x, t)$ in the surface water is observed, the model is over-parameterized because the concentration $C(x, t)$ is uniquely determined by the parameter ratios $\varepsilon_{S} / R_{H}^{2}$, $h_{s} / R_{H}$ and $d_{s} / R_{H}$. As we vary $R_{H}$ while keeping these ratios constant, the concentration profile in the sediment, $C_{s}(x, \hat{z}, t)$, does not change as a function of the scaled coordinate $\hat{z}=z / R_{H}$. Consequently, the right-hand side of equation (1),

$$
\frac{J(x, t)}{R_{H}}=\left.\frac{\varepsilon_{s}}{R_{H}^{2}} \frac{\partial C_{s}(x, \hat{z}, t)}{\partial \hat{z}}\right|_{\hat{z}=0}
$$


remains the same because $x$ and $t$ are not scaled. This implies that the interfacial diffusivity, $\varepsilon_{s}$, and the attenuation depth, $h_{s}$, cannot uniquely be determined by inverse modeling of solute BTCs in the water column unless the value of the hydraulic radius, $R_{H}$, is independently estimated. Alternatively, the tracer concentration would have to be measured also in the sediment bed at known depth, with careful assessment of the representativity of the samples in heterogeneous sediments.

The additional source of equifinality comes from the partially equivalent effect of in-stream dispersion, represented by the parameter $D$, and hyporheic mixing, represented by the parameters $\varepsilon_{s}$ and $h_{s}$. This can be shown analytically by looking at the behavior of the solutions of the model equations at large times, or numerically by imposing one of the model parameters and calibrating the remaining parameters to fit the same BTC. Appendix B presents an asymptotic solution for the concentration in the water column in the case of a semi-infinite bed with negligible underflow velocity, and shows that there are infinite sets of values for the parameters $A, D, \varepsilon_{s} / R_{H}^{2}$ and $h_{s} / R_{H}$, that produce approximately the same concentration distribution at large times. The conditions for equifinality of two different sets of parameter values, $\left(A, D, \varepsilon_{s} / R_{H}^{2}, h_{s} / R_{H}\right)$ and $\left(A^{\prime}, D^{\prime}, \varepsilon_{s}^{\prime} / R_{H}^{\prime 2}\right.$, $h_{s}^{\prime} / R_{H}^{\prime}$ ), for a given $\varepsilon_{s}^{\prime} / R_{H}^{\prime 2}$, are expressed by equations (B.7)-(B.9), which can be taken as valid for

$$
\frac{\varepsilon_{s} t}{h_{s}^{2}} \gtrsim \tau_{e}^{*}
$$

where $\tau_{e}^{*} \approx 780$. Note that the latter condition imposes a limit on the validity of the equifinality relationships (B.7-B.9) as a function of the hyporheic mixing scale, $h_{s}^{2} / \varepsilon_{s}$. If the model is calibrated using experimental data, the time $t$ in equation (16) should be taken as the starting time $t_{\min }$ of the BTC at which the measured concentration becomes distinguishable from the background noise, and therefore condition (16) provides an upper bound on the range of $h_{s}^{2} / \varepsilon_{s}$ for which a non-unique representation of the BTC is possible if the surface transport parameters $U=Q / A$ and $D$ are not known.

Numerical experiments show that equifinal sets of parameter values can be found also outside of the range of validity of equations (B.7-B.9). To illustrate this with a numerical experiment, the best-fit parameters $A, D$, and $h_{s} / R_{H}$ for the breakthrough curve of the Yarqon tracer test presented in section 4.1 were determined for different values of $\varepsilon_{s} / R_{H}^{2}$. Starting from the parameter values in Table 3 with $d_{s}=\infty$, the value of $\varepsilon_{s} / R_{H}^{2}$ was changed from $10^{-5}$ to $10^{2} \mathrm{~s}^{-1}$ and the remaining parameters $D, h_{s}$ and $A$ were cal- 
ibrated so as to minimize the RMSE between the simulated BTC and the observations. The resulting parameters are shown in Figure 5 as a function of $\varepsilon_{s} / R_{H}^{2}$. The continuous curves in the graph represent the parameters obtained by numerical best-fitting, whereas the dashed curves represent the parameters derived from the asymptotic analysis presented in Appendix B. The figure also shows the behavior of the RMSE. In the flat part of the RMSE curve the quality of the fit is the same, and on visual inspection the numerically generated BTCs appear to perfectly overlap with each other. For smaller values of $\varepsilon_{s} / R_{H}^{2}$, the RMSE increases, and the numerically generated BTCs gradually diverge. As $\varepsilon_{s} / R_{H}^{2}$ decreases below $10^{-4} \mathrm{~s}^{-1}$, the RMSE increases, and the simulated BTCs diverge. The differences first become visible in the initial rising limb of the BTC, and gradually grow to affect the concentration at increasingly larger times as $\varepsilon_{S} / R_{H}^{2}$ decreases. This means that the rising limb of the BTC is less affected by model equifinality than the tail. Figure 5 also shows that, although the validity of the approximations presented in Appendix B is restricted by condition (16) with $\tau_{e}^{*} \approx 780$, equifinal sets of parameter values exist over a broader range of $h_{s}^{2} / \varepsilon_{s}$. In this case, for $\varepsilon_{s} t_{\min } / h_{s}^{2}>40$ the RMSE becomes practically constant as a function of $\varepsilon_{S} / R_{H}^{2}$ and equal to its lowest value. Note that the equifinal relationships for $A$ and $h_{s}$ expressed by equations (B.7)-(B.8) can be taken as valid within the same range, whereas the best-fit longitudinal dispersion coefficient, $D$, significantly diverges from (B.9) for $\varepsilon_{s} t_{\min } / h_{s}^{2}<780$.

\section{Conclusions}

A physically based 1-D solute transport model was presented that represents hyporheic mixing as a diffusion process exponentially attenuated with depth from the sedimentwater interface. This parameterization is consistent with experimental results for turbulent flows over flat porous beds [Chandler et al., 2016], and with the exponential attenuation of the pumping-induced seepage velocity in case of bed form induced hyporheic exchange [Elliott and Brooks, 1997; Bottacin-Busolin and Marion, 2010]. Grant et al. [2018] used a depth-invariant effective diffusivity to represent the vertical mass transfer across the stream-water interface and found that, on average, the effective diffusivity for beds with bed forms and riffle-pool sequences is about 3.5 times higher than that predicted for flat sediment beds. This can be regarded as a small increase in comparison to the six-decade variability in the effective diffusivity associated with changes in the bed permeability and shear velocity [Grant et al., 2018]. In this context, the Exponentially Attenuated Mixing 


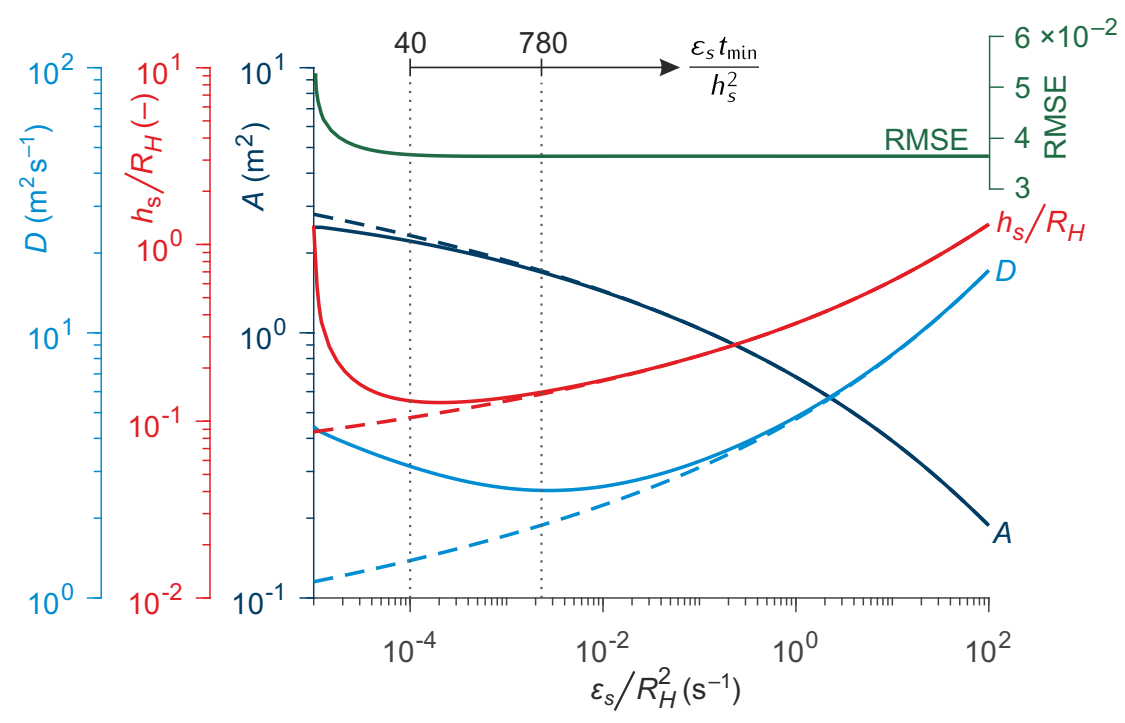

Figure 5. Best-fit parameters, $A, D$ and $h_{S} / R_{H}$, for the BTC of the Yarqon River (Figure 3c,d) as a function of $\varepsilon_{S} / R_{H}^{2}$. The continuous curves were obtained by numerical fitting, whilst the dashed curves were obtained from equations (B.7)-(B.9).

Model (EAMM) can be used to take into account the dispersive mixing associated with the laminarization and inertial penetration of stream turbulence into the streambed, as well as the vertical dispersive mixing induced by static and dynamic pressure variations over the bed surface.

The breakthrough curves (BTCs) generated by the EAMM can have exponential or power-law tempering at late times, with the latter associated with large vertical mixing time scales relative to the time of observation. In case of power-law behavior, the scaling exponent is found to be -2 in the case of exponentially attenuated mixing coefficient, and $-3 / 2$ in the uniform case. These scaling exponents are consistent with the latetime behavior of the residence time distributions of bed form induced hyporheic exchange [Bottacin-Busolin and Marion, 2010], which suggests that the applicability of the EAMM is not limited to flat sediment beds. Numerical tests show that the EAMM produces BTCs with superdiffusive scaling of the variance and non-decreasing skewness that are in agreement with experimental evidence from field tracer studies [González-Pinzón et al., 2013]. This was contrasted with the late-time Fickian behavior of a conventional two-storage zone model (TSZM) assuming first-order mass transfer with two storage domains of different cross-sectional area. The ability of the EAMM to represent experimental BTCs was tested using data from field and flume tracer experiments, and the results were compared with 
those of the TSZM. Whilst both the EAMM and the TSZM provided good approximations for the tracer BTCs observed in lowland rivers, the EAMM provided better fits of the BTCs measured in flume tracer experiments. These results support the conclusion that the EAMM can be used not only to describe hyporheic mixing, but also the combined effect of surface storage and hyporheic exchange.

Analysis of the EAMM equations and their solutions shows that there are two degrees of freedom in the estimation of the physical transport parameters from concentration distributions in the MFC. The implication of this result for tracer studies is that the physical parameters of hyporheic mixing cannot be determined solely from concentration measurements in the surface water unless the average flow velocity and flow depth are independently estimated. This is a consequence of the equifinality of the model parameters which results from two independent physical facts: first, the interfacial exchange flux depends only on the ratios between the hydraulic radius and the subsurface mixing parameters; second, the effect of hyporheic mixing becomes increasingly indistinguishable from surface mixing at late times. The first source of uncertainty can be mitigated by independent estimation of the reach-averaged hydraulic radius or purely by model calibration using a combination of surface and subsurface concentrations. The second source of uncertainty can be mitigated by independent estimation of the reach-averaged flow velocity, or by model calibration using multiple BTCs at different distances from the injection point. Given the heterogeneities that typically characterize natural watercourses, open questions concern the representativity of local geometry and concentration measurements, the relationship between spatial resolution and uncertainty in the estimated model parameters, and the optimal location of the measurements if uncertainty is to be minimized.

Alternatively, when predictive evaluation of hyporheic exchange and its effect on reach-scale solute transport is needed, the parameters of the exponentially attenuated mixing model can be estimated from empirical relationships [e.g., Chandler et al., 2016] or smaller-scale 2-D or 3-D simulations of turbulent flow over a porous bed. Potentially, the EAMM could also be coupled with hydrogeomorphic river network models such as NEXSS [Gomez-Velez and Harvey, 2014] to provide a multiscale representation of hyporheic exchange, ranging from grain-scale stream water-pore water interactions to basinscale advective flows induced by geomorphic features. 


\section{A Laplace Space Solution for 1-D Transport with Exponentially Attenuated Hy-} porheic Mixing

The solution to equations (1), (2) and (3) with the boundary conditions (4)-(5) can be derived by introducing the change of variable $\xi=x-u_{s} t$ and Laplace transforming with respect to $t$,

$$
\widetilde{C}(x, s)=\mathcal{L}[C(x, t) ; t \rightarrow s]=\int_{0}^{\infty} C(x, t) e^{-s t} d t
$$

The transformed equations are:

$$
\begin{aligned}
& s \widetilde{C}-C_{0}(\xi)+\left(U-u_{s}\right) \frac{\partial \widetilde{C}}{\partial \xi}-D \frac{\partial^{2} \widetilde{C}}{\partial \xi^{2}}=-\left.\frac{\varepsilon_{s}}{R_{H}} \frac{\partial \widetilde{C}_{s}}{\partial z}\right|_{z=0} \\
& s \widetilde{C}_{s}-\frac{\partial}{\partial z}\left[\left(\varepsilon_{\infty}+\left(\varepsilon_{s}-\varepsilon_{\infty}\right) e^{\frac{z}{h_{s}}}\right) \frac{\partial \widetilde{C_{s}}}{\partial z}\right]=0
\end{aligned}
$$

with the boundary conditions:

$$
\begin{aligned}
& \widetilde{C}(\xi, s)=\widetilde{C}_{s}(\xi, z=0, s) \\
& \left.\frac{\partial \widetilde{C}_{s}}{\partial z}\right|_{z=-d_{s}}=0
\end{aligned}
$$

Substitution of

$$
\zeta(z)=-\frac{\varepsilon_{s}-\varepsilon_{\infty}}{\varepsilon_{\infty}} e^{z / h_{s}} \quad, \quad \omega(\xi, z, s)=\left(\frac{\varepsilon_{s}-\varepsilon_{\infty}}{\varepsilon_{\infty}} e^{\frac{z}{h_{s}}}\right)^{-h_{s} \sqrt{\frac{s}{\varepsilon_{\infty}}}} \widetilde{C}_{s}(\xi, z, s)
$$

into (A.3) leads to the hypergeometric differential equation,

$$
\zeta(1-\zeta) \frac{\partial^{2} \omega}{\partial \zeta^{2}}+\left[\left(1+2 h_{s} \sqrt{\frac{s}{\varepsilon_{\infty}}}\right)-2\left(1+h_{s} \sqrt{\frac{s}{\varepsilon_{\infty}}}\right) \zeta\right] \frac{\partial \omega}{\partial \zeta}-h_{s} \sqrt{\frac{s}{\varepsilon_{\infty}}}\left(1+h_{s} \sqrt{\frac{s}{\varepsilon_{\infty}}}\right) \omega=0
$$

which has general solution [Polyanin and Zaitsev, 2017]

$$
\begin{aligned}
\omega(\xi, z, s)= & B_{1}(\xi, s) \zeta^{-2 h_{s} \sqrt{\frac{s}{\varepsilon_{\infty}}}}{ }_{2} F_{1}\left(1-h_{s} \sqrt{\frac{s}{\varepsilon_{\infty}}} ;-h_{s} \sqrt{\frac{s}{\varepsilon_{\infty}}} ; 1-2 h_{s} \sqrt{\frac{s}{\varepsilon_{\infty}}} ; \zeta\right)+ \\
& B_{2}(\xi, s)_{2} F_{1}\left(1+h_{s} \sqrt{\frac{s}{\varepsilon_{\infty}}} ; h_{s} \sqrt{\frac{s}{\varepsilon_{\infty}}} ; 1+2 h_{s} \sqrt{\frac{s}{\varepsilon_{\infty}}} ; \zeta\right)
\end{aligned}
$$

where $B_{1}$ and $B_{2}$ are arbitrary functions of $\xi$ and $s$. Substituting (A.6) into (A.8) and taking into account that $\widetilde{C}_{s}$ depends on $\xi$ only through condition (A.4), the general solution to the linear homogeneous equation (A.3) can be expressed as

$$
\widetilde{C}_{s}(\xi, z, s)=\left[a_{1}(s) \eta_{1}(z, s)+a_{2}(s) \eta_{2}(z, s)\right] \widetilde{C}(\xi, s)
$$


where

$$
\begin{aligned}
& \eta_{1}(z, s)=\left(\frac{\varepsilon_{s}-\varepsilon_{\infty}}{\varepsilon_{\infty}} e^{\frac{z}{h_{s}}}\right)^{-h_{s} \sqrt{\frac{s}{\varepsilon_{\infty}}}}{ }_{2} F_{1}\left(1-h_{s} \sqrt{\frac{s}{\varepsilon_{\infty}}} ;-h_{s} \sqrt{\frac{s}{\varepsilon_{\infty}}} ; 1-2 h_{s} \sqrt{\frac{s}{\varepsilon_{\infty}}} ;-\frac{\left(\varepsilon_{s}-\varepsilon_{\infty}\right) e^{\frac{z}{h_{s}}}}{\varepsilon_{\infty}}\right) \\
& \eta_{2}(z, s)=\left(\frac{\varepsilon_{s}-\varepsilon_{\infty}}{\varepsilon_{\infty}} e^{\frac{z}{h_{s}}}\right)^{h_{s} \sqrt{\frac{s}{\varepsilon_{\infty}}}}{ }_{2} F_{1}\left(1+h_{s} \sqrt{\frac{s}{\varepsilon_{\infty}}} ; h_{s} \sqrt{\frac{s}{\varepsilon_{\infty}}} ; 1+2 h_{s} \sqrt{\frac{s}{\varepsilon_{\infty}}} ;-\frac{\left(\varepsilon_{s}-\varepsilon_{\infty}\right) e^{\frac{z}{h_{s}}}}{\varepsilon_{\infty}}\right)
\end{aligned}
$$

and where $a_{1}$ and $a_{2}$ are arbitrary functions of $s$. Here ${ }_{2} F_{1}$ is the Gauss hypergeometric function, defined as

$$
{ }_{2} F_{1}(a ; b ; c ; z)=\sum_{n=0}^{\infty} \frac{(a)_{n}(b)_{n}}{(c)_{n}} \frac{z^{n}}{n !}
$$

where $(\cdot)_{n}$ is the Pochhammer symbol. The functions $a_{1}$ and $a_{2}$ are determined by imposing the boundary conditions (A.4) and (A.5):

$$
\begin{aligned}
& a_{1}(s)=\frac{\eta_{2, z}^{\prime}\left(-d_{s}, s\right)}{\eta_{1}(0, s) \eta_{2, z}^{\prime}\left(-d_{s}, s\right)-\eta_{1, z}^{\prime}\left(-d_{s}, s\right) \eta_{2}(0, s)} \\
& a_{2}(s)=\frac{-\eta_{1, z}^{\prime}\left(-d_{s}, s\right)}{\eta_{1}(0, s) \eta_{2, z}^{\prime}\left(-d_{s}, s\right)-\eta_{1, z}^{\prime}\left(-d_{s}, s\right) \eta_{2}(0, s)}
\end{aligned}
$$

where:

$$
\begin{aligned}
\eta_{1, z}^{\prime}(z, s)=\frac{\partial \eta_{1}}{\partial z}(z, s) & =-\eta_{1}(z, s) \sqrt{\frac{s}{\varepsilon_{\infty}}}+\frac{\left(\varepsilon_{s}-\varepsilon_{\infty}\right)\left(1-h_{s} \sqrt{\frac{s}{\varepsilon_{\infty}}}\right) e^{\frac{z}{h_{s}}} \sqrt{s}}{\varepsilon_{\infty}^{3 / 2}\left(1-2 h_{s} \sqrt{\frac{s}{\varepsilon_{\infty}}}\right)}\left(\frac{\varepsilon_{s}-\varepsilon_{\infty}}{\varepsilon_{\infty}} e^{\frac{z}{h_{s}}}\right)^{-h_{s} \sqrt{\frac{s}{\varepsilon_{\infty}}}} \\
& \times{ }_{2} F_{1}\left(1-h_{s} \sqrt{\frac{s}{\varepsilon_{\infty}}} ; 2-h_{s} \sqrt{\frac{s}{\varepsilon_{\infty}}} ; 2-2 h_{s} \sqrt{\frac{s}{\varepsilon_{\infty}}} ;-\frac{\left(\varepsilon_{s}-\varepsilon_{\infty}\right) e^{\frac{z}{h_{s}}}}{\varepsilon_{\infty}}\right) \\
\eta_{2, z}^{\prime}(z, s)=\frac{\partial \eta_{2}}{\partial z}(z, s) & =\eta_{2}(z, s) \sqrt{\frac{s}{\varepsilon_{\infty}}}-\frac{\left(\varepsilon_{s}-\varepsilon_{\infty}\right)\left(1+h_{s} \sqrt{\frac{s}{\varepsilon_{\infty}}}\right) e^{\frac{z}{h_{s}}} \sqrt{s}}{\varepsilon_{\infty}^{3 / 2}\left(1+2 h_{s} \sqrt{\frac{s}{\varepsilon_{\infty}}}\right)}\left(\frac{\varepsilon_{s}-\varepsilon_{\infty}}{\varepsilon_{\infty}} e^{\frac{z}{h_{s}}}\right)^{h_{s} \sqrt{\frac{s}{\varepsilon_{\infty}}}} \\
& \times{ }_{2} F_{1}\left(1+h_{s} \sqrt{\frac{s}{\varepsilon_{\infty}}} ; 2+h_{s} \sqrt{\frac{s}{\varepsilon_{\infty}}} ; 2+2 h_{s} \sqrt{\frac{s}{\varepsilon_{\infty}}} ;-\frac{\left(\varepsilon_{s}-\varepsilon_{\infty}\right) e^{\frac{z}{h_{s}}}}{\varepsilon_{\infty}}\right)
\end{aligned}
$$

The mass flux at the sediment-water interface is

$$
\left.\varepsilon_{s} \frac{\partial \widetilde{C}_{s}}{\partial z}\right|_{z=0}=\varepsilon_{s}\left[a_{1}(s) \eta_{1, z}^{\prime}(0, s)+a_{2}(s) \eta_{2, z}^{\prime}(0, s)\right] \widetilde{C}(\xi, s)
$$

Substituting into (A.2), the concentration in the water column can be expressed as

$$
\widetilde{C}(\xi, s)=\widetilde{C}_{w}(\xi, v(s))
$$

where $\widetilde{C}_{w}(\xi, s)$ is the Laplace transform of the solution of the classic advection-dispersion equation $(\mathrm{ADE})$ for the initial concentration $C_{0}(\xi)$, and

$$
v(s)=s+\frac{\varepsilon_{s}}{R_{H}}\left[a_{1}(s) \eta_{1, z}^{\prime}(0, s)+a_{2}(s) \eta_{2, z}^{\prime}(0, s)\right]
$$


is a frequency variable. For an instantaneous injection of mass $M, C_{0}(x)=(M / A) \delta(x)$, where $\delta(x)$ is the Dirac delta function, the solution to the advection-diffusion equation is given by

$$
\widetilde{C}_{w}(x, s)=\frac{M \exp \left[\frac{x U}{2 D}\left(1-\sqrt{1+\frac{4 D s}{U^{2}}}\right)\right]}{A \sqrt{U^{2}+4 D s}}
$$

For $z \rightarrow-\infty$, we recognize that $\eta_{1}(z, s) \rightarrow \infty$ and $\eta_{2}(z, s) \rightarrow 0$. Thus, for an infinitely deep bed,

$$
\widetilde{C}_{s}(\xi, z, s)=\frac{\eta_{2}(z, s)}{\eta_{2}(0, s)} \widetilde{C}(\xi, s)
$$

and therefore

$$
\left.\frac{\varepsilon_{s}}{R_{H}} \frac{\partial \widetilde{C}_{s}}{\partial z}\right|_{z=0}=\frac{\varepsilon_{s}}{R_{H}} \frac{\eta_{2, z}^{\prime}(z, s)}{\eta_{2}(0, s)} \widetilde{C}(\xi, s)
$$

As a special case, if $\varepsilon_{\infty}=0$, the solution to (A.3) is still given by (A.9) with coefficients (A.13)-(A.14) and

$$
\begin{aligned}
& \eta_{1}(z, s)=-I_{1}\left(2 h_{s} e^{-\frac{z}{2 h_{s}}} \sqrt{\frac{s}{\varepsilon_{s}}}\right) h_{s} e^{-\frac{z}{2 h_{s}}} \sqrt{\frac{s}{\varepsilon_{s}}} \\
& \eta_{2}(z, s)=2 K_{1}\left(2 h_{s} e^{-\frac{z}{2 h_{s}}} \sqrt{\frac{s}{\varepsilon_{s}}}\right) h_{s} e^{-\frac{z}{2 h_{s}}} \sqrt{\frac{s}{\varepsilon_{s}}}
\end{aligned}
$$

where $I_{1}(\cdot)$ and $K_{1}(\cdot)$ are the modified Bessel functions of the first and second kind, respectively. The derivatives $\eta_{1, z}^{\prime}$ and $\eta_{2, z}^{\prime}$ are given by

$$
\begin{aligned}
\eta_{1, z}^{\prime}(z, s)=\frac{1}{2}\left\{I_{1}\left(2 h_{s} e^{-\frac{z}{2 h_{s}}} \sqrt{\frac{s}{\varepsilon_{s}}}\right)+\right. & {\left[I_{0}\left(2 h_{s} e^{-\frac{z}{2 h_{s}}} \sqrt{\frac{s}{\varepsilon_{s}}}\right)\right.} \\
& \left.\left.+I_{2}\left(2 h_{s} e^{-\frac{z}{2 h_{s}}} \sqrt{\frac{s}{\varepsilon_{s}}}\right)\right] h_{s} e^{-\frac{z}{2 h_{s}}} \sqrt{\frac{s}{\varepsilon_{s}}}\right\} e^{-\frac{z}{2 h_{s}}} \sqrt{\frac{s}{\varepsilon_{s}}} \\
\eta_{2, z}^{\prime}(z, s)=-\left\{K_{1}\left(2 h_{s} e^{-\frac{z}{2 h_{s}}} \sqrt{\frac{s}{\varepsilon_{s}}}\right)-\right. & {\left[K_{0}\left(2 h_{s} e^{-\frac{z}{2 h_{s}}} \sqrt{\frac{s}{\varepsilon_{s}}}\right)\right.} \\
+ & \left.\left.K_{2}\left(2 h_{s} e^{-\frac{z}{2 h_{s}}} \sqrt{\frac{s}{\varepsilon_{s}}}\right)\right] h_{s} e^{-\frac{z}{2 h_{s}}} \sqrt{\frac{s}{\varepsilon_{s}}}\right\} e^{-\frac{z}{2 h_{s}}} \sqrt{\frac{s}{\varepsilon_{s}}}
\end{aligned}
$$

The solution can be further simplified for a bed of infinite depth, $d_{s}=\infty$. With reference to (A.9), in this case $a_{1}=0$ and $a_{2}(s)=1 / \eta_{2}(0, s)$; hence

$$
\widetilde{C}_{s}(\xi, z, s)=\frac{\eta_{2}(z, s)}{\eta_{2}(0, s)} \widetilde{C}(\xi, s)=\frac{e^{-\frac{z}{h_{s}}} K_{1}\left(2 h_{s} e^{-\frac{z}{2 h_{s}}} \sqrt{\frac{s}{\varepsilon_{s}}}\right)}{K_{1}\left(2 \sqrt{\frac{s}{\varepsilon_{s}}}\right)} \widetilde{C}(\xi, s)
$$

Finally, if the velocity in the subsurface is non-zero, $u_{s} \neq 0$, then

$$
C(x, z, t)=\mathcal{L}^{-1}\left[\widetilde{C}\left(x-u_{s} t, s\right) ; s \rightarrow t\right]
$$

where $\mathcal{L}^{-1}$ denotes the Bromwich integral. In the simulations presented in this paper, the inverse transformation (A.28) was performed numerically using de Hoog et al.'s algorithm [1982]. 


\section{B Equifinality Relationships at Large Times}

The equivalence conditions for the EAMM parameters in the asymptotic limit of large times can be derived by taking a series expansion of the Laplace-transformed concentration and equating terms of the same order in the Laplace variable $s$ as $s \rightarrow 0$. The derivations that follow are limited to the case of zero subsurface velocity, $u_{s}=0$, negligible mixing at large depth, $\varepsilon_{\infty}=0$, and sediment bed of infinite depth, $d_{s}=\infty$, for which the frequency variable $v(s)$ can be approximated as follows:

$$
\begin{aligned}
v(s) & =s-\frac{\varepsilon_{s}}{2 h_{s} R_{H}}\left\{1-h_{s} \sqrt{\frac{s}{\varepsilon_{s}}}\left[\frac{K_{0}\left(2 h_{s} \sqrt{\frac{s}{\varepsilon_{s}}}\right)+K_{2}\left(2 h_{s} \sqrt{\frac{s}{\varepsilon_{s}}}\right)}{K_{1}\left(2 h_{s} \sqrt{\frac{s}{\varepsilon_{s}}}\right)}\right]\right\} \\
& \approx v_{a}(s)=s-\left[2 \gamma+\log \left(\frac{h_{s}^{2} s}{\varepsilon_{s}}\right)\right] \frac{h_{s}}{R_{H}} s
\end{aligned}
$$

where $\gamma$ is the Euler-Mascheorni constant. From numerical tests, the truncation error of approximation (B.1) is lower than $1 \%$ if

$$
\frac{h_{s}^{2}|s|}{\varepsilon_{s}}<0.00128
$$

For an instantaneous mass release at $t=0$, combing (A.18) with $C_{w}$ (A.20) and (B.1) and expanding to second order in $s$ gives:

$$
\begin{gathered}
\widetilde{C}(x, s)=\frac{M}{Q}\left[1-\frac{1}{U^{2}}(2 D+U x) v_{a}(s)+\frac{1}{2 U^{4}}\left(12 D^{2}+6 D U x+U^{2} x^{2}\right) v_{a}(s)^{2}\right]+O\left(s^{3}\right) \\
=\frac{M}{Q}\left\{1-\frac{2 D+U x}{U^{2}} \frac{h_{s}}{R_{H}} s \log (s)-\frac{2 D+U x}{U^{2}}\left[1-2 \gamma \frac{h_{s}}{R_{H}}-\frac{h_{s}}{R_{H}} \log \left(\frac{h_{s}^{2}}{\varepsilon_{s}}\right)\right] s\right. \\
\left.+\frac{12 D^{2}+6 D U x+U^{2} x^{2}}{2 U^{4}}\left(\frac{h_{s}}{R_{H}}\right)^{2} s^{2} \log ^{2}(s)\right\}+O\left[s^{2} \log \left(\frac{h_{s}^{2} s}{\varepsilon_{s}}\right)\right]
\end{gathered}
$$

where $O(\cdot)$ means "order of". Equating terms of the same order in $s$ for two alternative sets of parameter values $\left(A, D, \varepsilon_{s}, h_{s}, R_{H}\right)$ and $\left(A^{\prime}, D^{\prime}, \varepsilon_{s}^{\prime}, h_{s}^{\prime}, R_{H}^{\prime}\right)$ gives the following conditions:

$$
\begin{aligned}
A^{2}(2 D+U x) \frac{h_{s}}{R_{H}} & =A^{\prime 2}\left(2 D^{\prime}+U^{\prime} x\right) \frac{h_{s}^{\prime}}{R_{H}^{\prime}} \\
A^{2}(2 D+U x) \frac{h_{s}}{R_{H}}\left[\frac{R_{H}}{h_{s}}-2 \gamma-\log \left(\frac{h_{s}^{2}}{\varepsilon_{s}}\right)\right] & =A^{\prime 2}\left(2 D^{\prime}+U^{\prime} x\right) \frac{h_{s}^{\prime}}{R_{H}^{\prime}}\left[\frac{R_{H}^{\prime}}{h_{s}^{\prime}}-2 \gamma-\log \left(\frac{h_{s}^{\prime 2}}{\varepsilon_{s}^{\prime}}\right)\right] \\
A^{4}\left(12 D^{2}+6 D U x+U^{2} x^{2}\right)\left(\frac{h_{s}}{R_{H}}\right)^{2} & =A^{\prime 4}\left(12 D^{\prime 2}+6 D^{\prime} U^{\prime} x+U^{\prime 2} x^{2}\right)\left(\frac{h_{s}^{\prime}}{R_{H}^{\prime}}\right)^{2}
\end{aligned}
$$

For a given parameter set $\left(A^{\prime}, D^{\prime}, \varepsilon_{s}^{\prime}, h_{s}^{\prime}, R_{H}^{\prime}\right)$, Equations (B.5-B.6) constitute a system of equations with two degrees of freedom that can be used to determine the equivalent parameter set $\left(A, D, \varepsilon_{s}, h_{s}, R_{H}\right)$. For example, if $\varepsilon_{s}$ and $R_{H}$ are given, the equifinal parame- 
ters $A, D$ and $h_{s}$ are:

$$
\begin{aligned}
& h_{s}=R_{H}\left[2 W\left(\frac{R_{H}^{\prime} e^{\frac{R_{H}^{\prime}}{2 h_{s}^{\prime}}}}{2 h_{s}^{\prime}} \sqrt{\frac{\varepsilon_{s}^{\prime} R_{H}^{2}}{\varepsilon_{S} R_{H}^{\prime 2}}}\right)\right]^{-1} \\
& A=A^{\prime} \frac{h_{s}^{\prime}}{h_{S}}=2 A^{\prime} \frac{R_{H}^{\prime}}{R_{H}} \frac{h_{s}^{\prime}}{R_{H}^{\prime}} W\left(\frac{R_{H}^{\prime} e^{\frac{R_{H}^{\prime}}{2 h_{s}^{\prime}}}}{2 h_{s}^{\prime}} \sqrt{\frac{\varepsilon_{s}^{\prime} R_{H}^{2}}{\varepsilon_{s} R_{H}^{\prime 2}}}\right) \\
& D=D^{\prime} \frac{h_{s}}{h_{s}^{\prime}}=D^{\prime} \frac{R_{H}}{R_{H}^{\prime}} \frac{R_{H}^{\prime}}{h_{s}^{\prime}}\left[2 W\left(\frac{R_{H}^{\prime} e^{\frac{R_{H}^{\prime}}{2 h_{s}^{\prime}}}}{2 h_{s}^{\prime}} \sqrt{\frac{\varepsilon_{s}^{\prime} R_{H}^{2}}{\varepsilon_{S} R_{H}^{\prime 2}}}\right)\right]^{-1}
\end{aligned}
$$

where $W(\cdot)$ is the Lambert $W$ function. Note that condition (B.2) provides a limit for the applicability of the relations above. Substitution of the frequency variable $s$ with $1 / t$, leads to (16) with $\tau_{e}^{*} \approx 780$.

\section{Acknowledgments}

All data used in this paper is properly cited and referred to in the reference list. The computer code implementing the exponentially attenuated mixing model (EAMM) can be downloaded from www.github.com/andreabottacin/EAMM. The author would like to thank Dr. Ricardo González-Pinzón and two anonymous reviewers for their useful comments on an earlier version of the manuscript.

\section{References}

Bencala, K. E., and R. A. Walters (1983), Simulation of solute transport in a mountain pool-and-riffle stream: A transient storage model, Water Resour. Res., 19(3), 718-724, doi:10.1029/WR019i003p00718.

Beven, K. (2006), A manifesto for the equifinality thesis, J. Hydrol., 320(1), 18-36, doi: 10.1016/j.jhydrol.2005.07.007.

Boano, F., C. Camporeale, R. Revelli, and L. Ridolfi (2006), Sinuosity-driven hyporheic exchange in meandering rivers, Geophys. Res. Lett., 33(18), L18,406, doi:10.1029/ 2006GL027630.

Boano, F., A. I. Packman, A. Cortis, R. Revelli, and L. Ridolfi (2007), A continuous time random walk approach to the stream transport of solutes, Water Resour. Res., 43(10), W10,425, doi:10.1029/2007WR006062.

Bottacin-Busolin, A. (2017), Non-Fickian dispersion in open-channel flow over a porous bed, Water Resour. Res., 53(8), 7426-7456, doi:10.1002/2016WR020348. 
Bottacin-Busolin, A., and A. Marion (2010), Combined role of advective pumping and mechanical dispersion on time scales of bed form-induced hyporheic exchange, Water Resour. Res., 46(8), W08,518, doi:10.1029/2009WR008892.

Bottacin-Busolin, A., G. Singer, M. Zaramella, T. J. Battin, and A. Marion (2009), Effects of Streambed Morphology and Biofilm Growth on the Transient Storage of Solutes, Environ. Sci. Technol., 43(19), 7337-7342, doi:10.1021/es900852w.

Bottacin-Busolin, A., A. Marion, T. Musner, M. Tregnaghi, and M. Zaramella (2011), Evidence of distinct contaminant transport patterns in rivers using tracer tests and a multiple domain retention model, Adv. Water Resour., 34(6), 737-746, doi:10.1016/j. advwatres.2011.03.005.

Breugem, W. P., B. J. Boersma, and R. E. Uittenbogaard (2006), The influence of wall permeability on turbulent channel flow, J. Fluid Mech., 562, 35-72, doi:10.1017/ S0022112006000887.

Briggs, M. A., M. N. Gooseff, C. D. Arp, and M. A. Baker (2009), A method for estimating surface transient storage parameters for streams with concurrent hyporheic storage, Water Resour. Res., 45(4), W00D27, doi:10.1029/2008WR006959.

Chandler, I. D., I. Guymer, J. M. Pearson, and R. van Egmond (2016), Vertical variation of mixing within porous sediment beds below turbulent flows, Water Resour. Res., 52(5), 3493-3509, doi:10.1002/2015WR018274.

Chen, X., M. B. Cardenas, and L. Chen (2018), Hyporheic Exchange Driven by ThreeDimensional Sandy Bed Forms: Sensitivity to and Prediction from Bed Form Geometry, Water Resources Research, 54(6), 4131-4149, doi:10.1029/2018WR022663.

Choi, J., J. W. Harvey, and M. H. Conklin (2000), Characterizing multiple timescales of stream and storage zone interaction that affect solute fate and transport in streams, $\mathrm{Wa}$ ter Resour. Res., 36(6), 1511-1518, doi:10.1029/2000WR900051.

Day, T. J. (1975), Longitudinal dispersion in natural channels, Water Resour. Res., 11(6), 909-918, doi:10.1029/WR011i006p00909.

de Hoog, F., J. Knight, and A. Stokes (1982), An Improved Method for Numerical Inversion of Laplace Transforms, SIAM J. Sci. and Stat. Comput., 3(3), 357-366, doi: $10.1137 / 0903022$.

de Lemos, M. J. S. (2005), Turbulent kinetic energy distribution across the interface between a porous medium and a clear region, Int. Commun. Heat Mass Transfer, 32(1-2), 107-115, doi:10.1016/j.icheatmasstransfer.2004.06.011. 
Deng, Z., L. Bengtsson, and V. P. Singh (2006), Parameter estimation for fractional dispersion model for rivers, Environ. Fluid Mech., 6(5), 451-475, doi:10.1007/ s10652-006-9004-5.

Drummond, J. D., T. P. Covino, A. F. Aubeneau, D. Leong, S. Patil, R. Schumer, and A. I. Packman (2012), Effects of solute breakthrough curve tail truncation on residence time estimates: A synthesis of solute tracer injection studies, J. Geophys. Res., 117(G3), G00N08, doi:10.1029/2012JG002019.

Elliott, A. H., and N. H. Brooks (1997), Transfer of nonsorbing solutes to a streambed with bed forms: Theory, Water Resour. Res., 33(1), 123-136, doi:10.1029/96WR02784.

Gomez-Velez, J. D., and J. W. Harvey (2014), A hydrogeomorphic river network model predicts where and why hyporheic exchange is important in large basins, Geophys. Res. Lett., 41(18), 2014GL061,099, doi:10.1002/2014GL061099.

González-Pinzón, R., R. Haggerty, and M. Dentz (2013), Scaling and predicting solute transport processes in streams, Water Resour. Res., 49(7), 4071-4088, doi:10.1002/wrcr. 20280.

Gooseff, M. N., D. M. McKnight, R. L. Runkel, and J. H. Duff (2004), Denitrification and hydrologic transient storage in a glacial meltwater stream, McMurdo Dry Valleys, Antarctica, Limnol. Oceanogr., 49(5), 1884-1895, doi:10.4319/lo.2004.49.5.1884.

Gooseff, M. N., M. A. Briggs, K. E. Bencala, B. L. McGlynn, and D. T. Scott (2013), Do transient storage parameters directly scale in longer, combined stream reaches? Reach length dependence of transient storage interpretations, J. Hydrol., 483, 16-25, doi:10. 1016/j.jhydrol.2012.12.046.

Grant, S. B., M. J. Stewardson, and I. Marusic (2012), Effective diffusivity and mass flux across the sediment-water interface in streams, Water Resour. Res., 48(5), n/a-n/a, doi: 10.1029/2011WR011148.

Grant, S. B., J. D. Gomez-Velez, and M. Ghisalberti (2018), Modeling the Effects of Turbulence on Hyporheic Exchange and Local-to-Global Nutrient Processing in Streams, Water Resour. Res., 54(9), 5883-5889, doi:10.1029/2018WR023078.

Haggerty, R., S. A. McKenna, and L. C. Meigs (2000), On the late-time behavior of tracer test breakthrough curves, Water Resour. Res., 36(12), 3467-3479, doi:10.1029/ 2000WR900214.

Haggerty, R., S. M. Wondzell, and M. A. Johnson (2002), Power-law residence time distribution in the hyporheic zone of a 2nd-order mountain stream, Geophys. Res. Lett., 
29(13), 18-1-18-4, doi:10.1029/2002GL014743.

Higashino, M., J. J. Clark, and H. G. Stefan (2009), Pore water flow due to near-bed turbulence and associated solute transfer in a stream or lake sediment bed, Water Resour. Res., 45(12), doi:10.1029/2008WR007374.

Jackman, A. P., R. A. Walters, and V. C. Kennedy (1984), Transport and concentration controls for chloride, strontium, potassium and lead in Uvas Creek, a small cobble-bed stream in Santa Clara County, California, U.S.A.: 2. Mathematical modeling, J. Hydrol., 75(1), 111-141, doi:10.1016/0022-1694(84)90047-7.

Kelleher, C., T. Wagener, B. McGlynn, A. S. Ward, M. N. Gooseff, and R. A. Payn (2013), Identifiability of transient storage model parameters along a mountain stream, Water Resources Research, 49(9), 5290-5306, doi:10.1002/wrcr.20413.

Kelleher, C., A. Ward, J. L. A. Knapp, P. J. Blaen, M. J. Kurz, J. D. Drummond, J. P. Zarnetske, D. M. Hannah, C. Mendoza-Lera, N. M. Schmadel, T. Datry, J. Lewandowski, A. M. Milner, and S. Krause (2019), Exploring Tracer Information and Model Framework Trade-Offs to Improve Estimation of Stream Transient Storage Processes, Water Resources Research, 55(4), 3481-3501, doi:10.1029/2018WR023585.

Kelly, J. F., D. Bolster, M. M. Meerschaert, J. D. Drummond, and A. I. Packman (2017), FracFit: A robust parameter estimation tool for fractional calculus models, Water Resour. Res., 53(3), 2559-2567, doi:10.1002/2016WR019748.

Kiel, B. A., and B. M. Cardenas (2014), Lateral hyporheic exchange throughout the Mississippi River network, Nature Geoscience, 7(6), 413-417, doi:10.1038/ngeo2157.

Marion, A., and M. Zaramella (2005), Diffusive behavior of bedform-induced hyporheic exchange in rivers, J. Environ. Eng., 131(9), 1260-1266, doi:10.1061/(ASCE) 0733-9372(2005)131:9(1260).

Marion, A., M. Zaramella, and A. I. Packman (2003), Parameter Estimation of the Transient Storage Model for Stream-Subsurface Exchange, J. Environ. Eng., 129(5), 456463, doi:10.1061/(ASCE)0733-9372(2003)129:5(456).

Marion, A., A. I. Packman, M. Zaramella, and A. Bottacin-Busolin (2008a), Hyporheic flows in stratified beds, Water Resour. Res., 44(9), W09,433, doi:10.1029/ 2007WR006079.

Marion, A., M. Zaramella, and A. Bottacin-Busolin (2008b), Solute transport in rivers with multiple storage zones: The STIR model, Water Resour. Res., 44(10), W10,406, doi:10.1029/2008WR007037. 
Marzadri, A., D. Tonina, A. Bellin, G. Vignoli, and M. Tubino (2010), Semianalytical analysis of hyporheic flow induced by alternate bars, Water Resour. Res., 46(7), W07,531, doi:10.1029/2009WR008285.

Nordin, C. F., and B. M. Troutman (1980), Longitudinal dispersion in rivers: The persistence of skewness in observed data, Water Resour. Res., 16(1), 123-128, doi:10.1029/ WR016i001p00123.

O’Connor, B. L., and J. W. Harvey (2008), Scaling hyporheic exchange and its influence on biogeochemical reactions in aquatic ecosystems, Water Resour. Res., 44(12), doi:10. 1029/2008WR007160.

Packman, A. I., M. Salehin, and M. Zaramella (2004), Hyporheic exchange with gravel beds: Basic hydrodynamic interactions and bedform-induced advective flows, J. Hydraul. Eng., 130(7), 647-656, doi:10.1061/(ASCE)0733-9429(2004)130:7(647).

Polyanin, A. D., and V. F. Zaitsev (2017), Handbook of Ordinary Differential Equations: Exact Solutions, Methods, and Problems, 3 edition ed., Chapman and Hall/CRC, Boca Raton London New York.

Rana, S. M. M., D. L. Boccelli, D. T. Scott, and E. T. Hester (2019), Parameter uncertainty with flow variation of the one-dimensional solute transport model for small streams using Markov chain Monte Carlo, Journal of Hydrology, 575, 1145-1154, doi: 10.1016/j.jhydrol.2019.06.003.

Roche, K. R., G. Blois, J. L. Best, K. T. Christensen, A. F. Aubeneau, and A. I. Packman (2018), Turbulence Links Momentum and Solute Exchange in Coarse-Grained Streambeds, Water Resour. Res., 54(5), 3225-3242, doi:10.1029/2017WR021992.

Roche, K. R., A. Li, D. Bolster, G. J. Wagner, and A. I. Packman (2019), Effects of turbulent hyporheic mixing on reach-scale transport, Water Resour. Res., O(ja), doi: 10.1029/2018WR023421.

Schmadel, N. M., B. T. Neilson, J. E. Heavilin, D. K. Stevens, and A. Wörman (2014), The influence of spatially variable stream hydraulics on reach scale transient storage modeling, Water Resources Research, 50(12), 9287-9299, doi:10.1002/2014WR015440.

Sherman, T., K. R. Roche, D. H. Richter, A. I. Packman, and D. Bolster (2019), A Dual Domain stochastic lagrangian model for predicting transport in open channels with hyporheic exchange, Adv. Water Resour., 125, 57-67, doi:10.1016/j.advwatres.2019.01.007.

Storn, R., and K. Price (1997), Differential Evolution - A Simple and Efficient Heuristic for global Optimization over Continuous Spaces, Journal of Global Optimization, 11(4), 
341-359, doi:10.1023/A:1008202821328.

Tonina, D., and J. M. Buffington (2007), Hyporheic exchange in gravel bed rivers with pool-riffle morphology: Laboratory experiments and three-dimensional modeling, Water Resour. Res., 43(1), W01,421, doi:10.1029/2005WR004328.

Voermans, J. J., M. Ghisalberti, and G. N. Ivey (2017), The variation of flow and turbulence across the sediment-water interface, J. Fluid Mech., 824, 413-437, doi: 10.1017/jfm.2017.345.

Ward, A. S., R. A. Payn, M. N. Gooseff, B. L. McGlynn, K. E. Bencala, C. A. Kelleher, S. M. Wondzell, and T. Wagener (2013), Variations in surface water-ground water interactions along a headwater mountain stream: Comparisons between transient storage and water balance analyses, Water Resour. Res., 49(6), 3359-3374, doi:10.1002/wrcr.20148.

Wondzell, S. M. (2006), Effect of morphology and discharge on hyporheic exchange flows in two small streams in the Cascade Mountains of Oregon, USA, Hydrol. Process., 20(2), 267-287, doi:10.1002/hyp.5902.

Wörman, A., A. I. Packman, H. Johansson, and K. Jonsson (2002), Effect of flow-induced exchange in hyporheic zones on longitudinal transport of solutes in streams and rivers, Water Resour. Res., 38(1), 2-1-2-15, doi:10.1029/2001WR000769.

Zaramella, M., A. Marion, J. Lewandowski, and G. Nützmann (2016), Assessment of transient storage exchange and advection-dispersion mechanisms from concentration signatures along breakthrough curves, J. Hydrol., 538, 794-801, doi:10.1016/j.jhydrol.2016. 05.004 .

Zhou, D., and C. Mendoza (1993), Flow through Porous Bed of Turbulent Stream, Journal of Engineering Mechanics, 119(2), 365-383, doi:10.1061/(ASCE)0733-9399(1993)119: 2(365). 